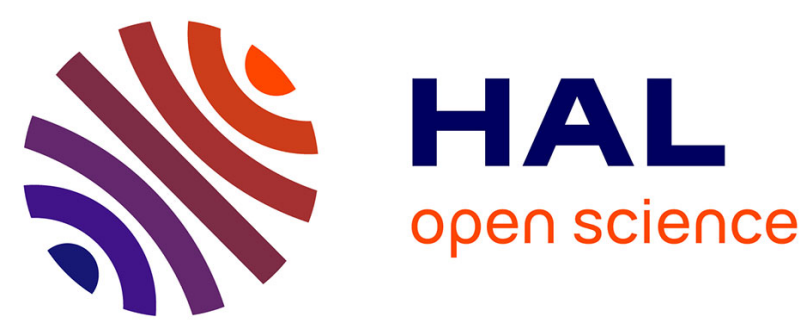

\title{
To vaccinate or not: Influenza virus and lung cancer progression
}

Mahmud Angrini, Aditi Varthaman, Ignacio Garcia-Verdugo, Jean-Michel Sallenave, Marco Alifano, Isabelle Cremer

\section{To cite this version:}

Mahmud Angrini, Aditi Varthaman, Ignacio Garcia-Verdugo, Jean-Michel Sallenave, Marco Alifano, et al.. To vaccinate or not: Influenza virus and lung cancer progression. Trends in Cancer, In press, 10.1016/j.trecan.2021.02.006 . hal-03170284

\section{HAL Id: hal-03170284 https://hal.sorbonne-universite.fr/hal-03170284}

Submitted on 16 Mar 2021

HAL is a multi-disciplinary open access archive for the deposit and dissemination of scientific research documents, whether they are published or not. The documents may come from teaching and research institutions in France or abroad, or from public or private research centers.
L'archive ouverte pluridisciplinaire $\mathbf{H A L}$, est destinée au dépôt et à la diffusion de documents scientifiques de niveau recherche, publiés ou non, émanant des établissements d'enseignement et de recherche français ou étrangers, des laboratoires publics ou privés. 


\title{
To vaccinate or not: Influenza virus and lung cancer progression
}

Mahmud Angrini ${ }^{1}$, Aditi Varthaman ${ }^{1}$, Ignacio Garcia-Verdugo², Jean-Michel Sallenave², Marco Alifano ${ }^{1,3}$ and Isabelle Cremer ${ }^{1}$

1. Centre de Recherche des Cordeliers, Sorbonne Universite, Inserm, Universite de Paris, Team Inflammation, complement and cancer, F-75006, Paris, France.

2. INSERM UMR1152; Laboratoire d’Excellence Inflamex, Université de Paris, France

3. Departement de chirurgie thoracique, Hopital Cochin AP-HP, Paris, France

Corresponding author: Isabelle Cremer, isabelle.cremer@sorbonne-universite.fr, INSERM U1138, Centre de Recherche des Cordeliers, 15 rue de l'Ecole de Médecine, 75006, Paris, France. Team inflammation, complement and cancer.

Keywords: Influenza virus, lung cancer, vaccine in cancer patients

\begin{abstract}
Influenza virus infection leads to severe and complicated disease particularly in lung cancer patients. It alters the tumor microenvironment (TME), which may potentiate lung cancer progression and disrupt responses to anti-tumoral treatments. Consequently, flu vaccination and antiviral treatments should be recommended to all lung cancer patients.
\end{abstract}


Influenza infection and cancer

Patients with cancer, and particularly those with lung cancer, are at a higher risk of morbidity and mortality from seasonal influenza. Monthly mortality rates for low and high flu months activity are 4.9 and $5.7 \%$ in lung cancer patients[1]. Additionally, the appearance of malignant cancer is linked to the prevalence of influenza infection that occurred one year before the detection of cancer[2] and a higher risk of developing lung cancer is related to cumulative exposure to the virus[3]. Recent studies have suggested that treatment with antivirals or vaccination against influenza may provide clinical benefit, including reduced hospitalization and reduced mortality, to patients with cancer [4] and current recommendations from Centers for disease control and prevention (CDC) and American Society for Clinical Oncology (ASCO) is to vaccinate cancer patients (https://www.cdc.gov/cancer/flu/basic-info.htm; https://ascopost.com/issues/september-25-2019/flu-vaccine-effectiveness-in-patients-withcancer). Mechanistically, vaccination may limit tumor progression by modulating anti-tumor immune responses. Herein, we stress the importance of vaccination against influenza in reducing lung cancer progression and incidence and discuss the mechanisms that may contribute to this reduced mortality.

Influenza induced inflammation and associated tumor microenvironment changes Cancer patients display higher persistent viral replication that may result in chronic inflammation, which is considered a hallmark of cancer and is strongly associated with tumor induction and/or progression. In lung cancer, chronic inflammation induced by influenza is dues to systemic and lung immunosuppression that results in lower viral clearance, and influenza specific RNA persistence in the lungs for more than one hundred days post-infection[5]. The prolonged 
infection and the resulting chronic inflammation lead to profound alteration of the tumor microenvironment (TME), which worsens tumor progression and disrupts responses to treatments.

Toll-like receptor (TLR) signaling in immune cells plays an essential role in immune surveillance and activation through recognition of pathogen-associated molecular patterns and stimulation of downstream proinflammatory cytokines. However, when expressed by cancer cells similar stimulation can promote tumor progression and survival [6]. Viral RNA detected by TLR7, which is highly expressed by lung tumor cells in many patients [6], activates interferon regulatory factor (IRF), nuclear factor-kappa B (NF-kB) and mitogen-activated protein kinase (MAPK) signaling pathways, followed by induction of inflammatory responses and the production of type I interferons (IFN-I), which promotes the expression of interferon-stimulated genes (ISGs). The persistence of viral RNA in the lungs following influenza infection may induce a prolonged stimulation of TLR7 in tumor cells - as we observed with repeated stimulation with synthetic TLR7 agonists- leading to overactivation of NF-kB and overexpression of $\mathrm{Bcl} 2$. This activation increases tumor cell survival and metastasis, and induces poor response to chemotherapy [6].

Influenza may also alter the anti-tumor immune response through $\mathrm{T}$ cell exhaustion and immunosuppression. Influenza infection of tumor bearing mice shunted anti-tumor $\mathrm{CD}^{+} \mathrm{T}^{\mathrm{T}}$ cells to the infection site, where they express high levels of the inhibitory receptor programmed cell death 1 (PD-1) resulting in inhibition of T cell function [7]. Influenza virus-infected cancer cells also downregulate class I major histocompatibility complex (MHC-I) expression, which promotes both tumor escape from cytotoxic $\mathrm{CD}^{+} \mathrm{T}$ cells leading to tumor progression and delayed viral clearance[8]. 
Influenza virus favors the establishment of an immunosuppressed TME, which promotes tumor growth. In a cohort of lung cancer patients from the cancer genome atlas (TCGA), a high ISGs score correlates with upregulation of multiple immunosuppressive factors including PD-L1, PDL2, IDO1 and LGALS9. This finding suggests that an additional mechanism by which infected lung cancer cells can evade immune control[9].

Viral persistence can also lead to development of resistance to anti-tumoral treatments via TME changes. Tumors resistant to immunotherapy exhibit sustained IFN-I signaling [9], similar to changes induced by viral persistence in the TME. Sustained IFN signaling in tumor cells induces STAT1-related epigenomic changes and increases the expression of PDL1 and NOS2 in antigen presenting cells, leading to increased resistance to immune checkpoint blockade of the PD1/PDL1 axis[10]. Moreover, a high ratio of ISGs in tumor cells versus ISGs in immune cells (T cells, NK cells, and macrophages) correlates with resistance to radiation, chemotherapy, and immune checkpoint blockade[11] (Figure 1).

\section{Antiviral treatment and influenza vaccine}

Considering the multiple, deleterious impacts of influenza infection on cancer progression, strategies to optimize influenza prevention among patients with cancer are highly warranted. Influenza vaccines and specific antiviral, flu medications are two suggested preventive therapeutics to be considered.

The optimal strategy to prevent influenza infection in cancer patients is vaccination. As the use of the live attenuated vaccine is contra-indicated in cancer patients, inactivated Influenza vaccines are the only available vaccine platform. Influenza vaccination correlates with reduced incidence of lung cancer as exampled in patients with chronic obstructive pulmonary disease 
(COPD), which is a well-known risk factor for lung cancer [12]. In patients undergoing curative surgery for solid cancers, giving a flu vaccine in the postoperative period results in less overall mortality and cancer-related mortality, compared with patients never receiving a vaccine [13]. Vaccination not only prevents influenza from modulating the TME directly, but it could also promote anti-tumor immunity in cancer patients. This has been demonstrated in murine studies where intra-tumoral flu vaccination reactivates $\mathrm{CD} 8^{+} \mathrm{T}$ cell-mediated antitumor immunity and sensitizes resistant tumors to immune checkpoint blockade (anti-PD-L1) [14].

However, few studies have shown that the immunogenicity of Influenza vaccines, based on clinical efficacy, is lower in cancer patients than in healthy individuals since it prevents infection in 10 to $60 \%$ cancer patients and in 70 to $90 \%$ in healthy people [4]. This suggests that healthy individuals, especially patient relatives and healthcare providers should also be encouraged to get the flu vaccine to prevent those with cancer from catching the virus. In order to improve influenza vaccine immunogenicity in lung cancer patients, different vaccination strategies should be investigated, such as using adjuvanted vaccine, two vaccine doses in the same influenza season, intranasal vaccine injections, different timing of vaccination, and high-dose vaccines. In addition, flu vaccination reduces the number of oncologic treatment interruptions compared to non-vaccinated cancer patients. Of note, receiving active chemotherapy doesn't change significantly the vaccine effectiveness among patients with solid tumor cancer [4]. Additionally, influenza vaccination in patients treated with immune checkpoint inhibitors seems safe and effective (https://www.esmo.org/oncology-news/influenza-vaccination-in-cancer-patientstreated-with-immune-checkpoint-inhibitors); However complementary studies that investigate 
the impact of Flu vaccination on targeted or immunotherapy should be performed to bring a strong conclusion.

For cancer patients who are not vaccinated, antiviral treatment such as neuraminidase inhibitor, (oseltamivir: Tamiflu), is recommended as early as possible for cancer patients with confirmed or suspected influenza, to reduce viral load and consequently limit the pro-tumoral effect of virus, with a probable impact on morbidity and mortality. However, because of viral persistence in cancer patients, the virus accumulate mutations during the course of infection, which leads to acquire resistance against antiviral treatments [15]. This emphasizes the recommendation of timely identifying resistant mutations that arise in the neuraminidase in infected cancer patients, and the importance to prevent the transmission of resistant influenza strains among cancer patients.

\section{Concluding remarks}

Influenza infection is more frequent, severe and complicated in cancer patients. Finding strategies to prevent and treat these infections can improve infection-related mortalities, and reduce the health, social and economic burden that arise from them. We must understand the impact of these infections on tumor progression, their short and long-term outcomes and define an optimal strategy to treat patients in such conditions. This is especially important since the same issue is currently seen with SARS-CoV-2 infection of lung cancer patients, that develop a more complicated and severe COVID-19 disease than the global population, with a probable similar pro-tumoral effect. For these patients, COVID-19 vaccination is therefore also highly recommended 
(https://perspectives.esmo.org/news/covid-19-vaccination-in-patients-with-cancer-esmoreleases-ten-statements; https://www.esmo.org/oncology-news/majority-of-patients-withcancer-are-willing-to-be-vaccinated-against-covid-19).

A better understanding of the mechanistic effects of influenza infection in different cells within the tumor-suppressive microenvironment may enable the development of novel therapeutic strategies to treat cancer patients. This knowledge would help adapt therapies in susceptible patients, for a better chance of success, and should be integrated into the arsenal of personalized therapies under development in lung cancer (Figure 2). Annual influenza vaccination, inexpensive and relatively safe preventive, in addition to early initiation of antiviral therapy should be highly recommended in this high-risk population, their relatives and healthcare providers. The capacity to improve the immunogenicity of vaccine is even expected to give a better protection in lung cancer patients.

Despite efforts to understand the impact of influenza infection in lung cancer patients, there are still many unanswered questions:

What are the short and long-term outcomes of influenza infection in lung cancer patients? How should physicians personalize lung cancer therapeutic protocols, in the case of influenza infection?

What is the best anti-influenza medication to be added to the therapy strategy of lung cancer patients? How can we avoid the development of drug resistance? Considering that influenza can cause viremia, does influenza infections influence the progression of other cancers? 


\section{Figure legends}

Figure 1: Summary of the impact of influenza infection on the tumor immune microenvironment. Influenza virus may infect tumor and immune cells leading to modulation of numerous immune characteristics: viral infection increases chemokine secretion (that are involved in immune cell migration), increases sustained type I IFNs and ISGs production (leading to resistance to treatments), increases reactive oxygen species (ROS) (involved in oxidative stress), increases immunosuppressive molecules (leading to immunosuppression), and increases inflammatory cytokines.

Figure 2: Consequences of influenza infection in patients with lung cancer. Influenza infection of lung tumor patients have multifactorial impacts in economy, health, flu vaccine strategies and anti-viral and anti-tumoral treatments.

\section{References}

1 Kinslow, C.J. et al. (2019) Influenza and mortality for non-small cell lung cancer. J. Clin. Oncol. 37, e13114-e13114

2 Inaida, S. and Matsuno, S. (2020) Previous Infection Positively Correlates to the Tumor Incidence Rate of Patients with Cancer. Cancer Immunol. Res. 8, 580-586

3 Weng, C.F. et al. (2019) Association between the risk of lung cancer and influenza: A populationbased nested case-control study. Int. J. Infect. Dis. 88, 8-13

$4 \quad$ Vollaard, A. et al. Influenza vaccination in adult patients with solid tumours treated with chemotherapy. , European Journal of Cancer, 76. 01-May-(2017) , Elsevier Ltd, 134-143

$5 \quad$ Keeler, S.P. et al. (2018) Influenza A Virus Infection Causes Chronic Lung Disease Linked to Sites of Active Viral RNA Remnants. J. Immunol. 201, 2354-2368

6 Chatterjee, S. et al. (2014) TLR7 Promotes Tumor Progression, Chemotherapy Resistance, and Poor Clinical Outcomes in Non-Small Cell Lung Cancer. Cancer Res. 74, 5008-5018

$7 \quad$ Kohlhapp, F.J. et al. (2016) Non-oncogenic Acute Viral Infections Disrupt Anti-cancer Responses and Lead to Accelerated Cancer-Specific Host Death. Cell Rep. 17, 957-965

8 Koutsakos, M. et al. (2019) Downregulation of MHC Class I Expression by Influenza A and B Viruses. Front. Immunol. 10, 1158 
9 Benci, J.L. et al. (2016) Tumor Interferon Signaling Regulates a Multigenic Resistance Program to Immune Checkpoint Blockade. Cell 167, 1540-1554.e12

10 Jacquelot, N. et al. (2019) Sustained Type I interferon signaling as a mechanism of resistance to PD-1 blockade. Cell Res. 29, 846-861

11 Benci, J.L. et al. (2019) Opposing Functions of Interferon Coordinate Adaptive and Innate Immune Responses to Cancer Immune Checkpoint Blockade. Cell 178, 933-948.e14

12 Chen, K.Y. et al. (2019) Effect of annual influenza vaccination on reducing lung cancer in patients with chronic obstructive pulmonary disease from a population-based cohort study. Med. (United States) 98,

13 Gögenur, M. et al. (2020) Association of postoperative influenza vaccine on overall mortality in patients undergoing curative surgery for solid tumors. Int. J. Cancer DOI: 10.1002/ijc.33340

14 Newman, J.H. et al. (2020) Intratumoral injection of the seasonal flu shot converts immunologically cold tumors to hot and serves as an immunotherapy for cancer. Proc. Natl. Acad. Sci. U. S. A. $117,1119-1128$

15 Mitha, E. et al. (2019) Safety, Resistance, and Efficacy Results from a Phase Illb Study of Conventional- and Double-Dose Oseltamivir Regimens for Treatment of Influenza in Immunocompromised Patients. Infect. Dis. Ther. 8, 613-626 


\section{Influenza}

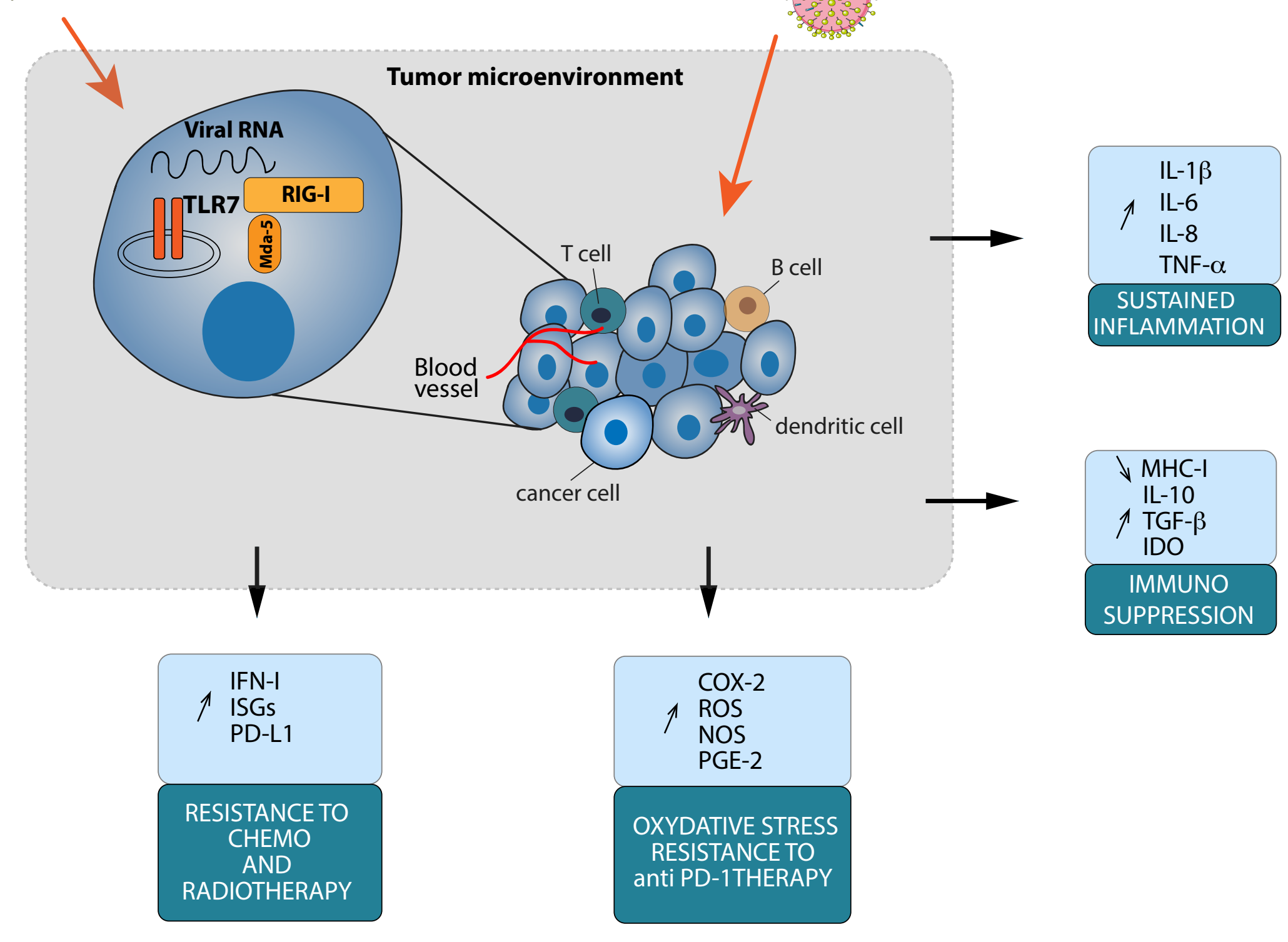




\section{Clinical Burden}

\section{Economic Burden}

-More hospitalization

-Higher hospitalization costs
-Longer and more complicated disease

-Prolonged viral shedding

-Higher rates of pneumonia

-Higher mortality

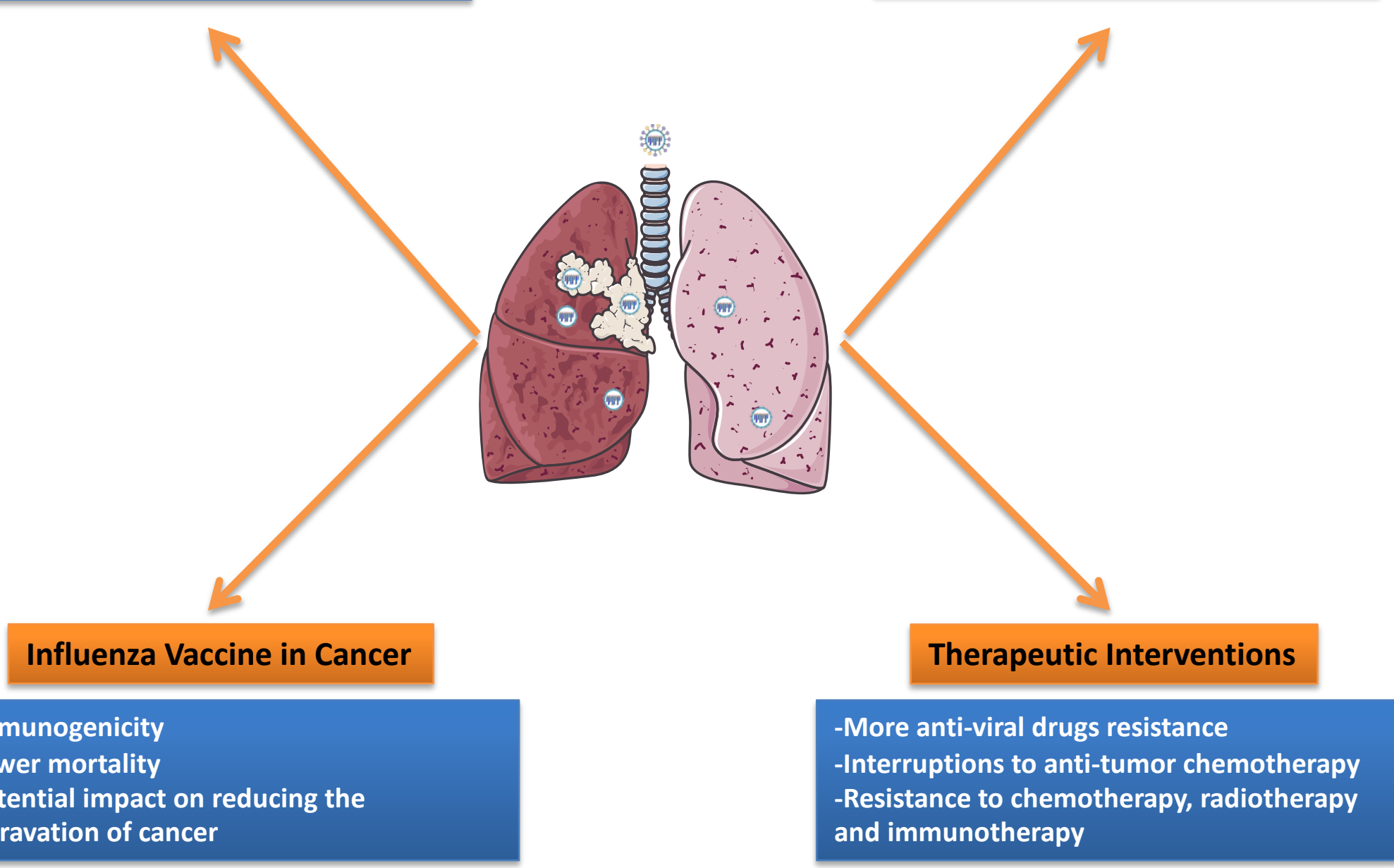

